journal club

\title{
Kombination toppt ASS-Monotherapie
}

Fragestellung: Ist niedrig dosiertes Rivaroxaban mit oder ohne Acetylsalicylsäure (ASS) einer ASS-Monotherapie bei Patienten mit Arteriosklerose und stabiler kardiovaskulärer Erkrankung überlegen?

Hintergrund: Die Sekundärprävention erfolgt bei Patienten mit stabiler koronarer Herzerkrankung (KHK) oder peripherer arterieller Verschlusskrankheit (PAVK) mit ASS oder Clopidogrel als Monotherapie. Das nicht Vitamin-K-abhängige orale Antikoagulans (NOAK) Rivaroxaban ist zur Schlaganfallprävention bei Patienten mit Vorhofflimmern und zur Prophylaxe sowie Therapie von tiefen Beinvenenthrombosen und Lungenembolien zugelassen. Die Autoren wollten untersuchen, ob Rivaroxaban in niedriger Dosis entweder als Monotherapie oder in Kombination mit ASS einer ASS-Monotherapie in der Sekundärprävention kardiovaskulärer Ereignisse überlegen ist.

Patienten und Methodik: Es handelte sich um eine doppelblinde Studie mit 27.395 Teilnehmern mit stabiler arteriosklerotischer, vaskulärer Erkrankung. Die Teilnehmer wurden in folgende drei Therapiegruppen eingeteilt: Die erste Gruppe erhielt täglich $2 \times 2,5 \mathrm{mg}$ Rivaroxaban plus $100 \mathrm{mg}$ ASS, die zweite Gruppe $2 \times 5 \mathrm{mg}$ Rivaroxaban und die dritte Gruppe $100 \mathrm{mg}$ ASS. Der primäre Endpunkt war die Kombination aus kardiovaskulärem Tod, Schlaganfall und Myokardinfarkt. Die Studie wurde vom Sicherheitskomitee wegen Überlegenheit der Therapiegruppe mit Rivaroxaban plus ASS nach 23 Monaten vorzeitig beendet.

Eikelboom JW, Connolly SJ, Bosch J et al. Rivaroxaban with or without aspirin in stable cardiovascular disease. $\mathrm{N}$ Engl J Med 2017; 377: 1319-30
Ergebnisse: Die Studienteilnehmer waren im Mittel 68 Jahre alt. $3,8 \%$ hatten in der Vergangenheit einen Schlaganfall erlitten und $62 \%$ einen Myokardinfarkt. $21 \%$ hatten eine Herzinsuffizienz. Bei $90 \%$ war eine KHK nachgewiesen und $27 \%$ litten unter einer PAVK.

In der Therapiegruppe, die Rivaroxaban plus ASS erhielt, trat bei 379 Patienten $(4,1 \%)$ der primäre Endpunkt auf, in der ASSMonotherapiegruppe war das bei 496 Patienten (5,4\%; Hazard Ratio [HR] 0,76, p < 0,001) der Fall. Schwerwiegende Blutungskomplikationen traten unter der Kombinationstherapie signifikant häufiger auf (3,1\% vs. $1,9 \%$ bei Monotherapie, $\operatorname{HR} 1,70$, $\mathrm{p}<0,001)$. Es ergaben sich aber keine signifikanten Unterschiede für intrakranielle oder tödliche Blutungen zwischen den Therapiegruppen. Die Sterblichkeit betrug 3,4\% in der Kombinations- und 4,1\% in der ASS-Monotherapiegruppe (HR 0,82, $\mathrm{p}=0,01)$. Der primäre Endpunkt war mit $4,9 \%$ in der Rivaroxaban-Monotherapiegruppe nicht unterschiedlich zur ASS-Monotherapiegruppe mit 5,4\%. Schwerwiegende Blutungsereignisse gab es bei 255 Patienten $(2,8 \%)$ mit Rivaroxaban-Monotherapie und bei 170 Patienten (1,9\%) mit ASS-Monotherapie (HR $1,51, \mathrm{p}=0,001)$. Die Zahl der Schlaganfälle war gering. Es zeigte sich aber eine signifikante Reduktion der Rate ischämischer Insulte mit 68 versus 91 für die Kombination von Rivaroxaban plus ASS, verglichen mit Rivaroxaban allein (HR 0,51). Rivaroxaban als Monotherapie führte ebenfalls zu signifikant weniger ischämischen Insulten mit 91 versus 132 bei ASS-Monotherapie (HR 0,69). Zerebrale Blutungen traten häufiger bei RivaroxabanMonotherapie auf mit 27 versus 10 bei ASS-Monotherapie und 15 bei Kombinationstherapie.

Schlussfolgerungen: Bei stabiler arteriosklerotischer vaskulärer Erkrankung reduziert die Kombination von täglich $2 \times 2,5$ mg Rivaroxaban plus 100 mg ASS sowohl kardiovaskuläre Ereignisse als auch die Sterblichkeit. Jedoch ist das Risiko schwerwiegender Blutungsereignisse signifikant erhöht. Die Wirkung der Rivaroxaban-Monotherapie unterschied sich nicht von der ASS-Monotherapie. Die Rivaroxaban-Monotherapie führte aber zu einem signifikanten Anstieg der Blutungskomplikationen.

\section{- Kommentar von Hans Christoph Diener, Essen}

\section{Interessant wird die Umsetzung des neuen Therapieprinzips werden}

Die COMPASS-Studie zeigte eine 24\%-ige Reduktion vaskulärer Endpunkte für die Kombination von niedrig dosiertem Rivaroxaban mit ASS und gleichzeitig eine $70 \%$-ige Zunahme schwerwiegender Blutungskomplikationen. Im klinischen Alltag ist es natürlich sehr schwierig, hier eine Güterabwägung zwischen Nutzen und Risiken vorzunehmen. Für die Beurteilung dieses Zielkonflikts ist es allerdings wichtig, dass die Rate an tödlichen und intrakraniellen Blutungen nicht signifikant unterschiedlich war. Eine Rivaroxaban-Monotherapie stellt keine therapeutische Alternative dar, da diese Therapie einer Behandlung mit ASS nicht überlegen war und ein höheres Blutungsrisiko hatte. Es wird interessant sein, in den nächsten Monaten und Jahren zu beobachten, ob im klinischen Alltag die Ergebnisse dieser gro- ßen und sehr gut durchgeführten Studie Eingang in die Praxis finden. Meine Voraussage ist, dass ähnlich wie beim Einsatz der NOAK zur Schlaganfallprävention bei Vorhofflimmern sehr wahrscheinlich Spezialisten wie Kardiologen und Angiologen das neue Therapieprinzip umsetzen werden, während wahrscheinlich viele Internisten und Hausärzte aus Angst vor der erhöhten Rate an Blutungskomplikationen bei ihrem bisherigen Ansatz mit einer Monotherapie mit Thrombozytenfunktionshemmern bleiben werden. Eine weitere Möglichkeit wäre, dass Patienten in einer potenziell vulnerablen Phase der Arteriosklerose mit der Kombinationstherapie behandelt werden und dass dann in der Langzeittherapie wieder auf eine Monotherapie mit Plättchenhemmern gewechselt wird. 